\title{
New Approach for Detection of Normal Alternative Splicing Events and Aberrant Spliceogenic Transcripts with Long-Range PCR and Deep RNA Sequencing
}

\author{
Vita Šetrajčič Dragoš ${ }^{1,2} @$, Vida Stegel ${ }^{1}$, Ana Blatnik ${ }^{3}$, Gašper Klančar ${ }^{1}$, Mateja Krajc ${ }^{3}$ \\ and Srdjan Novaković ${ }^{1,4, *}$ \\ 1 Department of Molecular Diagnostics, Institute of Oncology Ljubljana, SI-1000 Ljubljana, Slovenia; \\ vsetrajcic@onko-i.si (V.Š.D.); vstegel@onko-i.si (V.S.); gklancar@onko-i.si (G.K.) \\ 2 Biotechnical Faculty, University of Ljubljana, SI-1000 Ljubljana, Slovenia \\ 3 Cancer Genetics Clinic, Institute of Oncology Ljubljana, SI-1000 Ljubljana, Slovenia; ablatnik@onko-i.si (A.B.); \\ mkrajc@onko-i.si (M.K.) \\ 4 Faculty of Medicine, University of Ljubljana, SI-1000 Ljubljana, Slovenia \\ * Correspondence: snovakovic@onko-i.si; Tel.: +386-1-587-95-46
}

\section{check for}

updates

Citation: Dragoš, V.Š.; Stegel, V.; Blatnik, A.; Klančar, G.; Krajc, M.; Novaković, S. New Approach for Detection of Normal Alternative Splicing Events and Aberrant Spliceogenic Transcripts with Long-Range PCR and Deep RNA Sequencing. Biology 2021, 10, 706. https://doi.org/10.3390/ biology10080706

Academic Editor: Alper Kucukural

Received: 5 July 2021

Accepted: 21 July 2021

Published: 23 July 2021

Publisher's Note: MDPI stays neutral with regard to jurisdictional claims in published maps and institutional affiliations.

Copyright: (c) 2021 by the authors. Licensee MDPI, Basel, Switzerland. This article is an open access article distributed under the terms and conditions of the Creative Commons Attribution (CC BY) license (https:// creativecommons.org/licenses/by/ $4.0 /)$.
Simple Summary: RNA splicing defects, caused by genetic variants, are a common molecular mechanism of disease. To detect variants that cause splicing impairment, mRNA-based studies must be performed. Classical mRNA assays are time-consuming, which is why we have validated a new reliable straightforward approach to detect normal alternative splicing events and also splicing aberrations. Using our approach, we were able to reclassify three variants of uncertain significance in NBN and STK11 genes, which is of great importance for a proper clinical management of the patients.

\begin{abstract}
RNA sequencing is a promising technique for detecting normal and aberrant RNA isoforms Here, we present a new single-gene, straightforward 1-day hands-on protocol for detection of splicing alterations with deep RNA sequencing from blood. We have validated our method's accuracy by detecting previously published normal splicing isoforms of STK11 gene. Additionally, the same technique was used to provide the first comprehensive catalogue of naturally occurring alternative splicing events of the NBN gene in blood. Furthermore, we demonstrate that our approach can be used for detection of splicing impairment caused by genetic variants. Therefore, we were able to reclassify three variants of uncertain significance: NBN:c.584G>A, STK11:c.863-5_863-3delCTC and STK11:c.615G>A. Due to the simplicity of our approach, it can be incorporated into any molecular diagnostics laboratory for determination of variant's impact on splicing.
\end{abstract}

Keywords: splicing; RNA sequencing; alternative splicing; germline variant; spliceogenic variant; alternative transcript; splicing variant; DNA variant

\section{Introduction}

Alternative splicing is a process in which a single gene's pre-mRNA undergoes processing into multiple mature mRNA isoforms. Nearly all human multi-exon genes are involved in alternative splicing. For instance, $B R C A 1$ gene contains 23 exons, but 63 alternative splicing events are produced by wild-type allele [1]. Understanding the naturally occurring alternative splicing isoforms of clinically relevant genes is of great importance for correct interpretation of splicing assays. RNA splicing defects are a common molecular mechanism of disease, and there are studies demonstrating that RNA sequencing (RNAseq) considerably improves diagnostics yield [2-4]. In the study by Yamada et al., the authors showed that the detection rate of deleterious variants increased by $19 \%$ if combination of exome and transcriptome analysis was performed, compared with exome sequencing alone [2]. Similarly, Karam et al. reported that DNA sequencing (DNAseq) in combination 
with RNAseq improved clinical management of 1 in 43 patients in hereditary cancer syndromes [4]. Therefore, identification of splicing defects is of high importance to improve patient's management. Unfortunately, conventional RNA-based functional assays, such as minigene splicing assays, direct Sanger sequencing and capillary electrophoresis are labor intensive, difficult to interpret and often inconclusive [5]. An additional drawback of the above-mentioned conventional RNA assays is that the maximal fragment's length suitable for analysis is limited to approximately $1000 \mathrm{bp}$, which makes it impossible to investigate variants detected in long exons such as exon 10 in BRCA1 and exon 11 in BRCA2 gene. Therefore, to capture a variant's complete impact on splicing, whole gene RNA sequencing should be performed. With the development of next generation sequencing, targeted RNAseq or even whole transcriptome sequencing is nowadays technically feasible. However, for small diagnostic laboratories, whole transcriptome sequencing can be financially demanding, and it requires high computational power and storage capacity for RNAseq data analysis $[6,7]$.

Naturally occurring alternative splicing events must be determined by analyzing control samples alongside the patient sample to eliminate the possibility of misinterpreting the variant under investigation as spliceogenic. Previous studies have systematically determined alternative splicing events of genes associated with hereditary breast and/or ovarian cancer, such as BRCA1, BRCA2, PALB2 and STK11 [1,8,9]. However, genes associated with rare syndromes or genes with lower penetrance, e.g., $N B N$, remain less studied. Therefore, for small diagnostics laboratories dealing with large numbers of unclassified variants, it is crucial to develop a quick, easy, non-laborious and bioinformatically uncomplicated test for detecting splicing defects to minimize the number of variants of uncertain significance (VUS).

Consequently, the main purpose of our article is to describe a simple method for detecting variants that have an impact on splicing. Along with this, for the first time using our method, a list of alternative splicing events of the NBN gene is provided.

\section{Materials and Methods}

\subsection{Patient Samples}

A total of 5 patient blood samples and 6 unrelated control blood samples were collected into Tempus Blood RNA Tube (ThermoFisher, Waltham, MA, USA). Patient samples were carriers of the spliceogenic variants NF1:c.122A > T, NF1:c.7395-17T>G, NBN:c.584G>A, STK11:c.863-5_863-3delCTC and STK11:c.615G>A. Control samples were used for detection of alternative splicing events in STK11 and NBN genes. Total RNA was isolated from whole blood using Tempus ${ }^{\mathrm{TM}}$ Spin RNA Isolation Kit (ThermoFisher).

The present study was approved by the Institutional Review Board of the Institute of Oncology Ljubljana (permission no. OIRIEK00937) and by the National Medical Ethics Committee of Republic of Slovenia (permission no. 0120-339/2019/5). Individual patient consent was waived for this study, as it was a retrospective study, the research involved no risk to the subjects, and the institutional informed consent forms for treatment included consent for the use of patient's data, materials and/or test results for research purposes. All procedures followed in the present study were therefore in accordance with the ethical standards of the responsible committees on human experimentation (institutional and national) and the Helsinki Declaration of 1975, as revised in 2013.

\subsection{DNA Sequencing-DNAseq}

DNA sequencing was performed as previously described in Setrajcic Dragos et al., 2019 and Klancar et al., 2020 [10,11]. Control samples harboring only undoubtedly benign variants (described in Supplementary Table S2) in the coding region and $\pm 25 \mathrm{nt}$ of intronic sequence of STK11 and NBN gene were selected for alternative splicing isoform discovery. 


\subsection{RNA Sequencing-RNAseq}

cDNA synthesis was performed with SuperScript ${ }^{\mathrm{TM}}$ IV VILO $^{\mathrm{TM}}$ Master Mix (ThermoFisher) using 100ng of total RNA. Primers for genes STK11, NF1 and NBN were designed to flank $5^{\prime}$ and $3^{\prime}$ UTR. cDNA was amplified with long-range PCR using LongAmp ${ }^{\circledR}$ Taq $2 X$ Master Mix (New England Biolabs) (primer sequences and PCR conditions are described in the Supplementary Table S2).

PCR products were quantified with Qubit (ThermoFisher). Long-range PCR amplicons were used for further library preparation with Nextera XT according to manufactures' instructions (Illumina). The library was quantified with LabChip ${ }^{\circledR}$ GX Touch ${ }^{\mathrm{TM}}$ Nucleic Acid Analyzer (PerkinElmer). The library was paired-end sequenced $(2 \times 121$ cycles $)$ on NextSeq 550 (Illumina).

Raw data files (bcl) were converted to fastq files using bcl2fastq2 tool. FastQC tool was used to determine the quality of NGS data. STAR aligner 2.7.3a was used for alignment of NGS reads to hg19 genome assembly, with the following settings: -outFilterMultimapNmax 2 -outFilterMismatchNmax 20 -chimSegmentMin 0 [12] Samtools was used to create index bam (bam.bai) file [13]. All splicing events were obtained from OutSJ.tab file, produced by STAR. Sashimi plots were created using rmats2sashimi tool. Bioinformatic tools for splicing prediction NNSplice, MaxEntScan, Gene splicer, SpliceSiteFinder-like (included in Alamut visual software) and SpliceAI were used [14].

\subsection{Alternative Splicing Events Threshold}

Junctions covered with a minimum of 20 reads and present in at least two samples or previously published were considered as real splicing junctions. Junctions below the set threshold were regarded as sequencing artifacts and/or biological outliers.

\subsection{Identification of Splicing Aberrations Caused by Genetic Variant}

Genetic variants and alternative splicing events are described following HGVS nomenclature v19.01, where c.1 and r.1 are the A of the ATG translation initiation codon. Reference transcripts NM_000455.4, NM_002485.4 and NM_000267.3 for genes STK11, NBN and NF1 were used, respectively. Alternative splicing isoform was defined as any splice junction not defined in the above-mentioned reference transcripts. Splicing isoforms are described using symbols: $\Delta$ ((partial) exon skipping), $\mathbf{\nabla}$ (intron insertion), $\mathrm{p}$ (acceptor shift) and $\mathrm{q}$ (donor shift). If multiple cryptic exon inclusion events occurred within the same intron, subsequent letters were added to the event. For example, if three cryptic exon inclusion evets occurred between exons 4 and 5 , we described it as $\nabla 4 \mathrm{~A}, \mathbf{\nabla} 4 \mathrm{~B}, \mathbf{\nabla} 4 \mathrm{C}$.

\section{Results}

Here, we present a straightforward 1-day hands-on protocol for detection of splicing alterations in blood with deep RNAseq (cDNA seq), regardless of the gene in question. The test is based on long-range PCR with primers aligning to the $5^{\prime}$ UTR and $3^{\prime}$ UTR regions of the targeted cDNA. The long PCR amplicon is then fragmented with Nextera transposome and tagged with a universal overhang. Next generation sequencing (NGS) library is further prepared with Illumina's Nextera XT. Schematic representation of the novel RNAseq method is presented in Figure 1. 


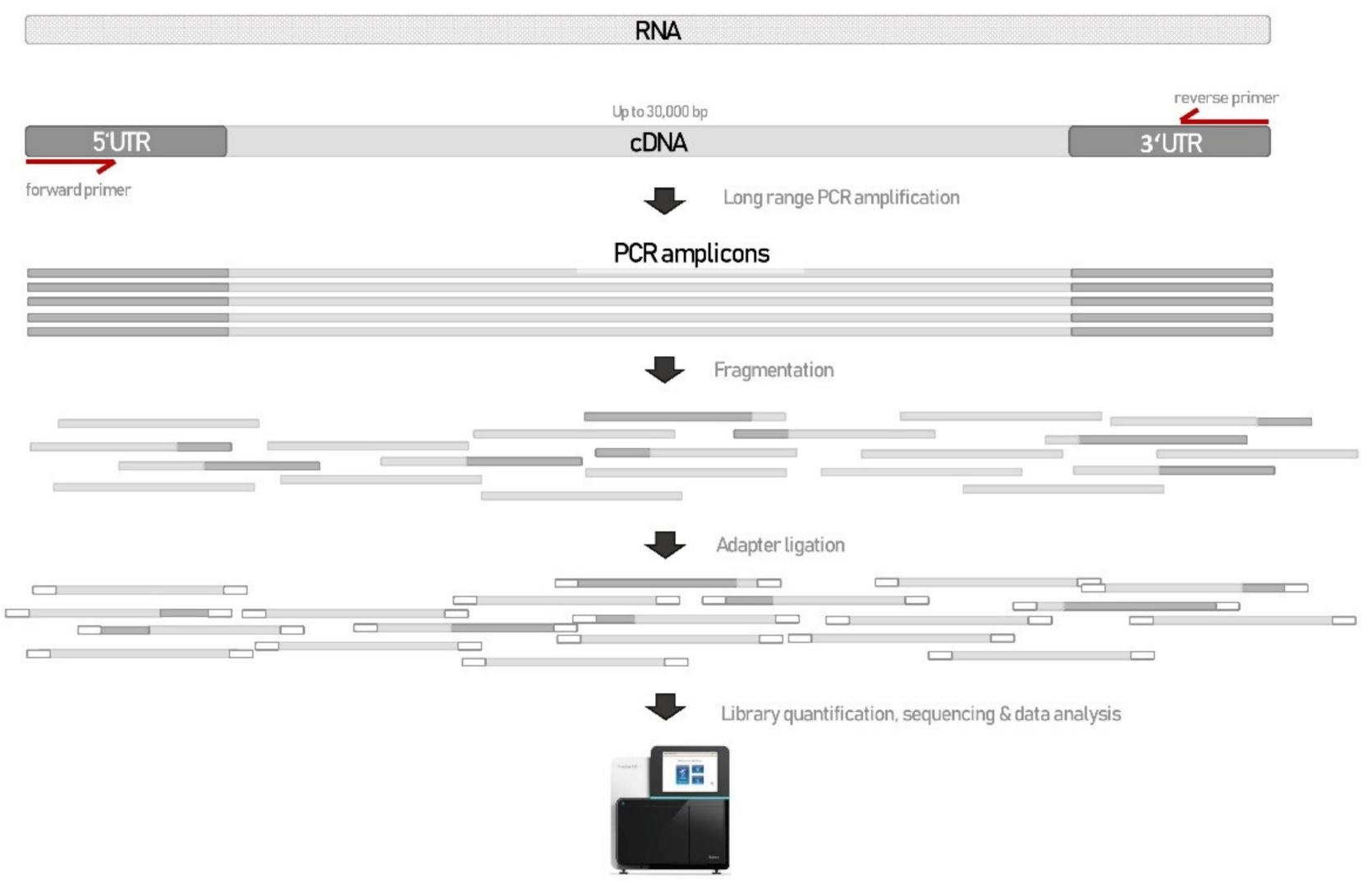

Figure 1. Schematic representation of novel approach used to detect splicing aberration with deep RNAseq.

\subsection{Method Confirmation-Alternative Splicing Events in STK11 Gene}

The first step in our study was to confirm that our new NGS library preparation and bioinformatics pipeline can reliably detect all major splicing events including exonic and intronic splice-site shift, cryptic exon inclusion and (multiple) exon skipping. Therefore, we decided to determine all naturally occurring splicing junctions of STK11 gene expressed in blood and compare our results with those previously identified by Brandão et al., 2019. In order to detect even less expressed events, we were aiming for coverage of canonical splice junctions above $100,000 \times$. We were able to detect $36 / 38(95 \%)$ of previously reported STK11 splicing junctions, missing one exon skipping and one multi exon skipping event. However, not all previously reported junctions reached our threshold (covered with at least 20 reads and present in at least 2 samples): one junction $\Delta 4-5$ was expressed extremely weakly in all 6 samples with the average of 6 reads, whereas junctions $\mathbf{\nabla} 1 \mathrm{H}, \mathbf{v} 1 \mathrm{I}$ and $\mathbf{\nabla} 7 \mathrm{q}$ were expressed in one sample only, but with a considerable number of reads spanning the junction: 116, 175 and 29 reads, respectively. We were unable to detect two previously published junctions of STK11 gene, $\Delta 2-5$ and $\Delta 7$. Hence, we aligned our data again to the sequence of the two known events and visually inspected the alignment. No reads mapped to those two events, suggesting they were indeed not present in our data. The splicing event $\Delta 7$ was however detected in a patient sample, which harbored a leaky splice-site variant STK11:c.863-5_863-3delCTC in intron 6, causing the exon 7 skipping. Four splicing events were predominantly expressed (with a percentage of reads $>1 \%$ ): $\mathbf{\nabla} 1 \mathrm{C}$, $\nabla 7 C, \nabla 7 D$ and $\Delta 9 q$, graphically presented in Figure 2 . All four events are predicted to create frameshifts and a premature stop codon, resulting in nonsense-mediated decay or nonfunctional protein. The highest expressed splicing event that can possibly retain protein function was $\Delta 2-3(0.37 \%)$, causing an in-frame deletion of amino acids 98-155. In addition, we detected 18 splicing events that have not been published before (Table 1), suggesting that our approach can be useful for detection of splicing events in STK11 gene. 


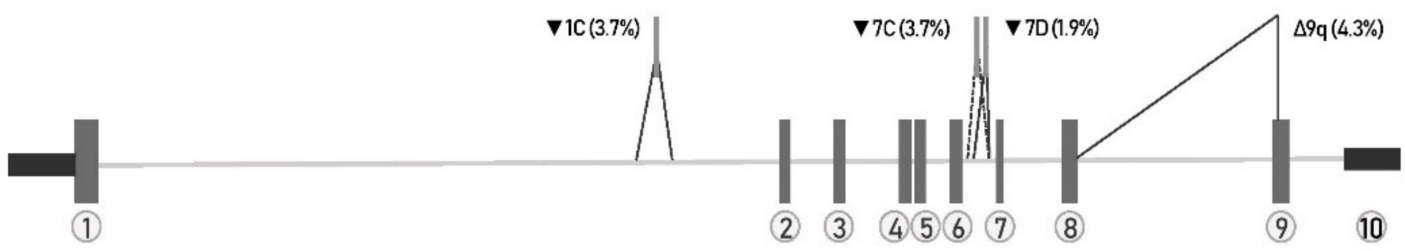

STK11 (NM_000455.4)

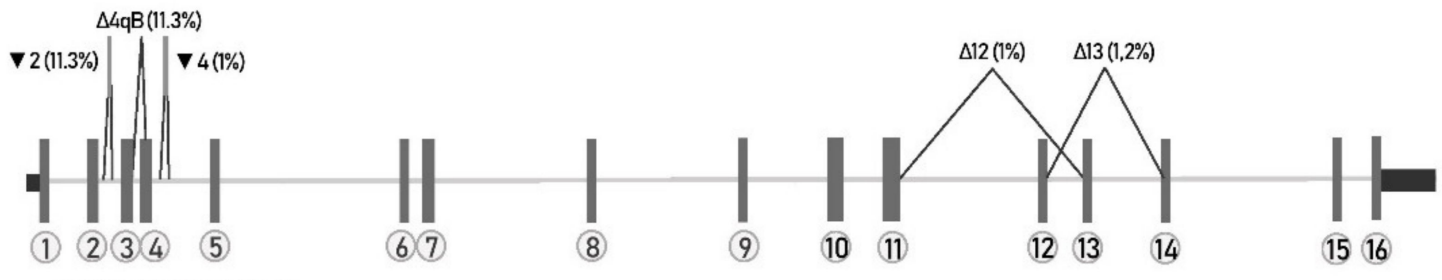

NBN (NM_002485.4)

Figure 2. Schematic representation of major STK11 and NBN alternative splicing events. Only splicing events with expression higher than $1 \%$ are shown in the image.

Table 1. Splicing events of STK11 gene detected by new RNAseq approach from control samples in comparison with data published by Brandão et al., 2019 [9].

\begin{tabular}{|c|c|c|c|c|c|c|}
\hline RNA Consequence & $\begin{array}{c}\text { Junction } \\
\text { Description }\end{array}$ & $\begin{array}{l}\text { Average Number } \\
\text { of Reads } \\
\text { Supporting the } \\
\text { Junction }(N=6)\end{array}$ & $\begin{array}{c}\text { Mean } \\
\text { Percentage of } \\
\text { Junction } \\
\text { Reads }+\end{array}$ & Biotype & $\begin{array}{l}\text { Percentage of } \\
\text { Samples with } \\
\text { Observed } \\
\text { Junction }\end{array}$ & $\begin{array}{c}\text { Detected } \\
\text { by } \\
\text { Brandão } \\
\text { et al., 2019 }\end{array}$ \\
\hline r.-272_-186del & $\Delta 5^{\prime} \mathrm{UTR}$ & 64 & 0.026 & terminal modification & 33 & no \\
\hline r.-245_-209del & $\Delta 5^{\prime} \mathrm{UTR}$ & 56 & 0.023 & terminal modification & 50 & no \\
\hline r.-258_-185del & $\Delta 5^{\prime} \mathrm{UTR}$ & 150 & 0.061 & terminal modification & 50 & no \\
\hline r.-323_597del & $\Delta 5^{\prime} \mathrm{UTR}$ & 86 & 0.029 & terminal modification & 33 & no \\
\hline r.290 291ins $290+2456 \quad 290+2554$ & $\nabla 1 \mathrm{~A}$ & 608 & 0.207 & cryptic exon inclusion & 67 & yes \\
\hline r.290_291ins290+5106_290+5326 & $\mathbf{\nabla} 1 \mathrm{~B}$ & 44 & 0.015 & cryptic exon inclusion & 50 & yes \\
\hline r.290_291ins291-2149_291-2019 & $\mathbf{\nabla} 1 \mathrm{C}$ & 10,912 & 3.717 & cryptic exon inclusion & 100 & yes \\
\hline r.290_291ins291-2038_291-102 & $\mathbf{\nabla} 1 \mathrm{D}$ & 70 & 0.024 & cryptic exon inclusion & 83 & yes \\
\hline r.290_291ins291-2897_291-2755 & $\nabla 1 E$ & 72 & 0.025 & cryptic exon inclusion & 33 & no \\
\hline r.290_291ins291-2149_291-1782 & $\boldsymbol{\nabla} 1 \mathrm{~F}$ & 105 & 0.071 & cryptic exon inclusion & 67 & no \\
\hline & $\nabla 1 G$ & 146 & 0.050 & cryptic exon inclusion & 100 & yes \\
\hline r.290_291ins290+114_290+190 † & $\mathbf{\nabla} 1 \mathrm{H}$ & 116 & 0.040 & cryptic exon inclusion & 17 & yes \\
\hline r.290_291ins291-2149_291-1324 & $\mathbf{v} 1 \mathrm{I}$ & 175 & 0.060 & cryptic exon inclusion & 17 & yes \\
\hline intron 1 junction & TI & 293,569 & 1 & intron 1 junction & 100 & yes \\
\hline r.374delinsAC & $\Delta 2 \mathrm{pA}$ & 405 & 0.141 & exonic donor shift & 100 & no \\
\hline r.374insA_375delG & $\Delta 2 \mathrm{pB}$ & 117 & 0.041 & exonic donor shift & 100 & no \\
\hline r.373_376del & $\Delta 2,3 \mathrm{q}$ & 274 & 0.096 & exonic acceptor shift & 100 & no \\
\hline r.291_464del & $\Delta 2-3$ & 1140 & 0.373 & multiple exon skipping & 100 & yes \\
\hline intron 2 junction & 1 & 286,038 & 1 & intron 2 junction & 100 & yes \\
\hline r.373_378del & $\Delta 3 q$ & 227 & 0.079 & exonic acceptor shift & 67 & no \\
\hline intron 3 junction & $l^{1}$ & 316,895 & / & intron 3 junction & 100 & yes \\
\hline r.465_597del & $\Delta 4$ & 532 & 0.186 & exon skipping & 100 & yes \\
\hline r.490_653del & $\Delta 4 \mathrm{p}, \Delta 5 \mathrm{q}$ & 67 & 0.022 & mixed & 50 & no \\
\hline r.465 $734 \mathrm{del} \S$ & $\Delta 4-5$ & 6 & 0.002 & multiple exon skipping & 100 & yes \\
\hline r.465_920del & $\Delta 4-7$ & 62 & 0.023 & multiple exon skipping & 100 & yes \\
\hline intron 4 junction & / & 254,161 & / & intron 4 junction & 100 & yes \\
\hline r.706_734del & $\Delta 5$ & 342 & 0.125 & exon skipping & 83 & yes \\
\hline r.734del & $\Delta 5 \mathrm{p}$ & 29 & 0.010 & exonic donor shift & 83 & no \\
\hline intron 5 junction & l & 295,148 & / & intron 5 junction & 100 & yes \\
\hline r.862del & $\Delta 6 \mathrm{p}$ & 36 & 0.015 & exonic donor shift & 100 & no \\
\hline r.862_863ins862+281_863-103 & v7A & 1545 & 0.661 & cryptic exon inclusion & 100 & yes \\
\hline r.862_863ins862+286_863-103 & $\nabla 7 \mathrm{~B}$ & 1218 & 0.521 & cryptic exon inclusion & 100 & yes \\
\hline r.862_863ins863-283_863-103 & $\mathbf{\nabla} 7 \mathrm{C}$ & 3230 & 1.382 & cryptic exon inclusion & 100 & yes \\
\hline r.862_863ins863-253_863-103 & $\mathbf{v} 7 \mathrm{D}$ & 4570 & 1.956 & cryptic exon inclusion & 100 & yes \\
\hline r.862_863ins863-195_863-103 & $\boldsymbol{\nabla} 7 \mathrm{E}$ & 186 & 0.079 & cryptic exon inclusion & 67 & no \\
\hline r.858_862del +r.862_863ins863-125_863-103 & $\nabla 7 \mathrm{~F}$ & 16 & 0.007 & mixed & 33 & no \\
\hline r.862_863ins863-126_863-103 & $\mathbf{v} 7 \mathrm{G}$ & 25 & 0.011 & cryptic exon inclusion & 33 & no \\
\hline r.862_863ins863-125_863-103 & $\mathbf{v} 7 \mathrm{H}$ & 202 & 0.087 & cryptic exon inclusion & 100 & no \\
\hline r.820_921ins921-34_921-1 & $\nabla 7 q$ & 29 & 0.012 & intronic acceptor shift & 17 & yes \\
\hline intron 6 junction & $j^{1}$ & 233,667 & / & intron 6 junction & 100 & yes \\
\hline r.920_921ins921-105_921-1 & $\nabla 8 \mathrm{qA}$ & 185 & 0.074 & intronic acceptor shift & 100 & yes \\
\hline r.920_921ins921-87_921-1 & $\mathbf{v} 8 \mathrm{qB}$ & 186 & 0.074 & intronic acceptor shift & 83 & yes \\
\hline intron 7 junction & / & 250,858 & $/$ & intron 7 junction & 100 & yes \\
\hline r.1180_1181ins1108+466_1108+600 & $\mathbf{\nabla} 8 \mathrm{~A}$ & 749 & 0.331 & cryptic exon inclusion & 100 & yes \\
\hline r.1180_1181ins_1180+1_1108+187 & $\nabla 8 p$ & 141 & 0.062 & intronic donor shift & 33 & no \\
\hline r.1180_1181ins1108+466_1109-641 & $\mathbf{\nabla} 8 \mathrm{~B}$ & 95 & 0.042 & cryptic exon inclusion & 50 & yes \\
\hline
\end{tabular}


Table 1. Cont.

\begin{tabular}{|c|c|c|c|c|c|c|}
\hline RNA Consequence & $\begin{array}{c}\text { Junction } \\
\text { Description }\end{array}$ & $\begin{array}{l}\text { Average Number } \\
\text { of Reads } \\
\text { Supporting the } \\
\text { Junction }(\mathrm{N}=6)\end{array}$ & $\begin{array}{c}\text { Mean } \\
\text { Percentage of } \\
\text { Junction } \\
\text { Reads } \dagger\end{array}$ & Biotype & $\begin{array}{l}\text { Percentage of } \\
\text { Samples with } \\
\text { Observed } \\
\text { Junction }\end{array}$ & $\begin{array}{c}\text { Detected } \\
\text { by } \\
\text { Brandão } \\
\text { et al., 2019 }\end{array}$ \\
\hline intron 8 junction & / & 226,042 & / & intron 8 junction & 100 & yes \\
\hline r.1109_1113del & $\Delta 9 q$ & 9881 & 4.371 & exonic acceptor shift & 100 & yes \\
\hline r. $1109+16 \mathrm{del}$ & $\Delta 9$ & 67 & 0.047 & exon skipping & 33 & yes \\
\hline intron 9 junction & / & 56,173 & / & intron 9 junction & 100 & yes \\
\hline
\end{tabular}

$\dagger —$ calculation method that determines the percentage of detected junctions, adapted from Davy et al., 2017; $\ddagger$ events detected in one sample only; §- events covered with less than 20 reads.

\subsection{Catalogue of Naturally Occurring Splicing Events in NBN Gene}

Once the new method was established, we examined the alternative splicing events in NBN gene, with an identical approach (Table 2). In the previous studies, 10 alternatively spliced isoforms have been identified with RT-PCR [15-17]. Using our approach, we were able to identify all 10 previously described alternative splicing events as well as 49 previously undescribed alternative splicing events. To our knowledge, this is the most extensive catalogue of naturally occurring alternative splicing events of the NBN gene. Altogether, we detected 59 alternative splicing events; $11(\Delta 2 q, \Delta 3-4, \Delta 4-5, \Delta 6-7, \Delta 12$, $\Delta 13 \mathrm{qA}, \Delta 13, \Delta 12-13, \Delta 14, \Delta 13-14, \Delta 12-14)$ were predicted to be in-frame deletions that can possibly rescue the protein function. In-frame deletions of exon $13(\Delta 13)$ and exon 12 $(\Delta 12)$ were expressed the highest, with $1.2 \%$ and $1 \%$, respectively. Only exons 8,10 and 11 were not a subject of exon skipping. In NBN gene, we were able to detect two splicing events affecting $3^{\prime} \mathrm{UTR}$ region, which might actually be alternative $3^{\prime} \mathrm{UTR}$ isoforms [18].The highest expressed alternative splicing event was cryptic exon inclusion $\mathbf{\nabla} 2(11.3 \%)$, which corresponds to exon 3 in NCBI reference sequence NM_001024688.2. Events present in more than $1 \%$ are depicted schematically in Figure 2. All splicing junctions produced by STAR aligner of both studied genes are listed in the Supplementary Table S1.

Table 2. Splicing events of NBN (NBS1) gene detected by new RNAseq approach from control samples.

\begin{tabular}{|c|c|c|c|c|c|c|}
\hline RNA Consequence & $\begin{array}{c}\text { Junction } \\
\text { Description }\end{array}$ & $\begin{array}{l}\text { Average Number } \\
\text { of Reads } \\
\text { Supporting the } \\
\text { Junction }(\mathrm{N}=6)\end{array}$ & $\begin{array}{c}\text { Mean } \\
\text { Percentage of } \\
\text { Junction } \\
\text { Reads }+\end{array}$ & Biotype & $\begin{array}{c}\text { Percentage of } \\
\text { Samples with } \\
\text { Observed } \\
\text { Junction }\end{array}$ & $\begin{array}{l}\text { Detected } \\
\text { by Varon } \\
\text { et al., } 2006\end{array}$ \\
\hline r.37_38ins37+466_37+648 & $\mathbf{\nabla} 1 \mathrm{~A}$ & 72 & 0.179 & cryptic exon inclusion & 50 & No \\
\hline r.37_38ins37+698_37+779 & $\nabla 1 B$ & 33 & 0.080 & cryptic exon inclusion & 50 & No \\
\hline intron 1 junction & l & 52,217 & / & intron 1 junction & 100 & No \\
\hline r.38 $40 \mathrm{del}$ & $\Delta 2 \mathrm{q}$ & 17 & 0.033 & exonic acceptor shift & 67 & No \\
\hline r.171_172ins $\overline{172}-479 \_172-430$ & $\mathbf{\nabla} 2$ & 12,105 & 11.297 & cryptic exon inclusion & 100 & yes \\
\hline r.171__172ins172-27_172-1 & $\mathbf{\nabla} 3 q$ & 50 & 0.070 & intronic acceptor shift & 83 & No \\
\hline r.171_172ins171+1_171+4 & $\nabla 4 \mathrm{p}$ & 35 & 0.048 & intronic donor shift & 100 & No \\
\hline intron 2 junction & I & 72,077 & / & intron 2 junction & 100 & No \\
\hline r.38_171del & $\Delta 2$ & 31 & 0.169 & exon skipping & 83 & No \\
\hline intron $\overline{3}$ junction & / & 115,981 & / & intron 3 junction & 100 & No \\
\hline r.172_320del & $\Delta 3-4$ & 21 & 0.020 & multiple exon skipping & 33 & No \\
\hline r.172_320del & $\Delta 3$ & 137 & 0.145 & exon skipping & 83 & No \\
\hline r.321_325del & $\Delta 4 \mathrm{qA}$ & 37 & 0.032 & exonic acceptor shift & 67 & No \\
\hline r.321_361del & $\Delta 4 \mathrm{qB}$ & 3341 & 2.881 & exonic acceptor shift & 100 & No \\
\hline r.172_361del NM_001024688.2 & & 31 & / & $\begin{array}{l}\text { exon skipping+exonic } \\
\text { acceptor shift }\end{array}$ & 83 & No \\
\hline r.172_361del & $\Delta 3+4 q C$ & 118 & 0.126 & $\begin{array}{l}\text { exon skipping+exonic } \\
\text { acceptor shift }\end{array}$ & 67 & No \\
\hline r.480_481ins480+306_480+395 & $\mathbf{\nabla} 4$ & 1294 & 1.041 & cryptic exon inclusion & 100 & No \\
\hline intron 4 junction & / & 138,006 & l & intron 4 junction & 100 & No \\
\hline r.38_480del & $\Delta 2-4$ & 24 & 0.023 & multiple exon skipping & 83 & No \\
\hline r.321_480del & $\Delta 4$ & 58 & 0.045 & exon skipping & 50 & No \\
\hline r.172_480del & $\Delta 3-4$ & 43 & 0.040 & multiple exon skipping & 33 & No \\
\hline r. $4 \overline{8} 1 \mathrm{del}$ & $\Delta 5 \mathrm{qB}$ & 22 & 0.016 & exonic acceptor shift & 100 & No \\
\hline intron 5 junction & $l^{1}$ & 135,653 & / & intron 5 junction & 100 & No \\
\hline r.481_584del & $\Delta 5$ & 891 & 0.651 & exon skipping & 100 & Yes \\
\hline r.321_584del & $\Delta 4-5$ & 161 & 0.116 & multiple exon skipping & 100 & Yes \\
\hline r.172_584del & $\Delta 3-5$ & 37 & 0.035 & multiple exon skipping & 33 & No \\
\hline r.38_584del & $\Delta 2-5$ & 164 & 0.174 & multiple exon skipping & 83 & No \\
\hline r.589del & $\Delta 6 \mathrm{qB}$ & 86 & 0.064 & exonic acceptor shift & 100 & No \\
\hline intron 6 junction & 1 & 139,974 & / & intron 6 junction & 100 & No \\
\hline r.585_702del & $\Delta 6$ & 75 & 0.054 & exon skipping & 50 & No \\
\hline r.703_820del & $\Delta 7 \mathrm{q}$ & 127 & 0.090 & exonic acceptor shift & 50 & Yes \\
\hline intron $\overline{7}$ junction & $/^{1}$ & 179,463 & / & intron 7 junction & 100 & No \\
\hline r.585_896del & $\Delta 6-7$ & 118 & 0.075 & multiple exon skipping & 83 & Yes \\
\hline r.481_896del & $\Delta 5-7$ & 202 & 0.224 & multiple exon skipping & 67 & No \\
\hline r.38_896del & $\Delta 2-7$ & 61 & 0.052 & multiple exon skipping & 67 & No \\
\hline r.897del & $\Delta 8 \mathrm{q}$ & 17 & 0.010 & exonic acceptor shift & 100 & No \\
\hline r.994_995ins994+1178_995-1769 & $\mathbf{\nabla} 8 \mathrm{~A}$ & 86 & 0.041 & cryptic exon inclusion & 67 & No \\
\hline
\end{tabular}


Table 2. Cont.

\begin{tabular}{|c|c|c|c|c|c|c|}
\hline RNA Consequence & $\begin{array}{c}\text { Junction } \\
\text { Description }\end{array}$ & $\begin{array}{l}\text { Average Number } \\
\text { of Reads } \\
\text { Supporting the } \\
\text { Junction }(N=6)\end{array}$ & $\begin{array}{c}\text { Mean } \\
\text { Percentage of } \\
\text { Junction } \\
\text { Reads }+\end{array}$ & Biotype & $\begin{array}{l}\text { Percentage of } \\
\text { Samples with } \\
\text { Observed } \\
\text { Junction }\end{array}$ & $\begin{array}{l}\text { Detected } \\
\text { by Varon } \\
\text { et al., } 2006\end{array}$ \\
\hline r.994_995ins995-1769_995-1604 & $\mathbf{\nabla} 8 \mathrm{~B}$ & 34 & 0.015 & cryptic exon inclusion & 33 & No \\
\hline intron 8 junction & l & 202,305 & / & intron 8 junction & 100 & No \\
\hline r.1124_1125ins1124+703_1124+760 & $\nabla 9$ & 391 & 0.220 & cryptic exon inclusion & 100 & Yes \\
\hline intron 9 junction & / & 181,550 & / & intron 9 junction & 100 & No \\
\hline r.995_1124del & $\Delta 9$ & 173 & 0.270 & exon skipping & 100 & No \\
\hline intron 10 junction & / & 127,561 & / & intron 10 junction & 100 & No \\
\hline r.1398del & $\Delta 11 \mathrm{qA}$ & 29 & 0.023 & exonic acceptor shift & 100 & No \\
\hline r.1398_1403del & $\Delta 11 \mathrm{qB}$ & 83 & 0.065 & exonic acceptor shift & 33 & No \\
\hline r.1398_1471del & $\Delta 11 \mathrm{qC}$ & 70 & 0.055 & exonic acceptor shift & 83 & No \\
\hline r.1845_1846ins1845+1521_1845+1597 & $\nabla 11$ & 22 & 0.030 & cryptic exon inclusion & 33 & No \\
\hline r.1845_1846ins1846-23_1846-1 & $\nabla 12 q$ & 68 & 0.052 & intronic acceptor shift & 83 & No \\
\hline intron 11 junction & / & 132,009 & / & intron 11 junction & 100 & No \\
\hline r.1846_1849del & $\Delta 12 q$ & 46 & 0.035 & exonic acceptor shift & 67 & No \\
\hline intron 12 junction & / & 128,083 & / & intron 12 junction & 100 & No \\
\hline r.1896_1914del & $\Delta 12 p$ & 152 & 0.118 & exonic donor shift & 100 & No \\
\hline r.1846 1914del & $\Delta 12$ & 894 & 1.030 & exon skipping & 100 & No \\
\hline r.1915_1932del & $\Delta 13 \mathrm{qA}$ & 316 & 0.247 & exonic acceptor shift & 100 & No \\
\hline r.1915_2009del & $\Delta 13 \mathrm{qB}$ & 114 & 0.089 & exonic acceptor shift & 67 & Yes \\
\hline intron 13 junction & 1 & 128,636 & / & intron 13 junction & 100 & No \\
\hline r.1915_2070del & $\Delta 13$ & 1576 & 1.228 & exon skipping & 100 & Yes \\
\hline r.1846 2070del & $\Delta 12-13$ & 96 & 0.074 & multiple exon skipping & 83 & No \\
\hline r.2184_2185ins2184+417_2184+464 & $\nabla 14 \mathrm{~A}$ & 59 & 0.042 & cryptic exon inclusion & 100 & No \\
\hline r.2184_2185ins $2184+1511 \_2184+1578$ & $\boldsymbol{\nabla} 14 \mathrm{~B}$ & 58 & 0.036 & cryptic exon inclusion & 67 & No \\
\hline r.2184_2185ins2185-735_2185-610 & $\mathbf{\nabla} 14 \mathrm{C}$ & 76 & 0.059 & cryptic exon inclusion & 83 & No \\
\hline r.2184_2185ins2185-718_2185-610 & $\nabla 14 \mathrm{D}$ & 184 & 0.149 & cryptic exon inclusion & 100 & Yes \\
\hline r.2184_2185ins2185-4_2185-1ins & $\mathbf{\nabla} 14 \mathrm{q}$ & 33 & 0.026 & intronic acceptor shift & 33 & No \\
\hline intron 14 junction & / & 123,705 & / & intron 14 junction & 100 & No \\
\hline r.2071_2184del & $\Delta 14$ & 98 & 0.077 & exon skipping & 100 & No \\
\hline r.1915_2184del & $\Delta 13-14$ & 45 & 0.073 & multiple exon skipping & 83 & No \\
\hline r.1846_2184del & $\Delta 12-14$ & 222 & 0.174 & multiple exon skipping & 100 & Yes \\
\hline intron 15 junction & / & 136,201 & / & intron 15 junction & 100 & No \\
\hline r.2185_2234del & $\Delta 15$ & 70 & 0.054 & exon skipping & 83 & No \\
\hline r.1915_2234del & $\Delta 13-15$ & 100 & 0.075 & multiple exon skipping & 100 & No \\
\hline r.*39_*541del & $\Delta 3^{\prime} \mathrm{UTR}$ & 93 & 0.068 & terminal modification & 67 & No \\
\hline r.2003_*1085del & $\Delta 13 p+\Delta 14-$ & 74 & 0.058 & $\begin{array}{c}\text { multiple exon } \\
\text { skipping+exonic donor } \\
\text { shift }\end{array}$ & 33 & No \\
\hline r. ${ }^{*} 1076 \_* 1143 \mathrm{del}$ & $\Delta 3^{\prime} \mathrm{UTR}$ & 27 & 0.020 & terminal modification & 33 & No \\
\hline
\end{tabular}

†-calculation method that determines the percentage of detected junctions, adapted from Davy et al., 2017.

\subsection{Detection of Known Spliceogenic Variants}

To verify that our method is capable of detecting an abnormal splicing pattern caused by a spliceogenic variant, we used our method to test two variants previously characterized as spliceogenic. Impact on splicing for both variants has been previously established with direct Sanger sequencing and capillary electrophoresis (CE) [10]. Variant 1 is NF1:c.122A $>$ T r.121_204del, which creates an exonic donor shift leading to deletion of 84 bp of exon $2(\Delta 2 q)$. Assessed by CE, NF1:c.122A $>$ T induced transcript represented $52 \%$ in comparison to $48 \%$ of full-length transcript. Variant 2 is NF1:c.7395-17T>G r.7394_7395ins7395-16_7395-1, which causes an acceptor shift and retention of last $16 \mathrm{bp}$ of intron 50 ( $\mathbf{\nabla} 50 \mathrm{p})$. Aberrant transcript was present in $17.3 \%$ determined by CE. Indeed, our new RNAseq protocol successfully identified the disruption of normal splicing and determined the exact splicing junction in both samples (Figure 3). Additionally, according to the new RNAseq method, the fraction of aberrant transcripts caused by NF1 variants was $45 \%$ and $19 \%$ for NF1:c.122A $>$ T and NF1:c.7395-17T>G, respectively. 

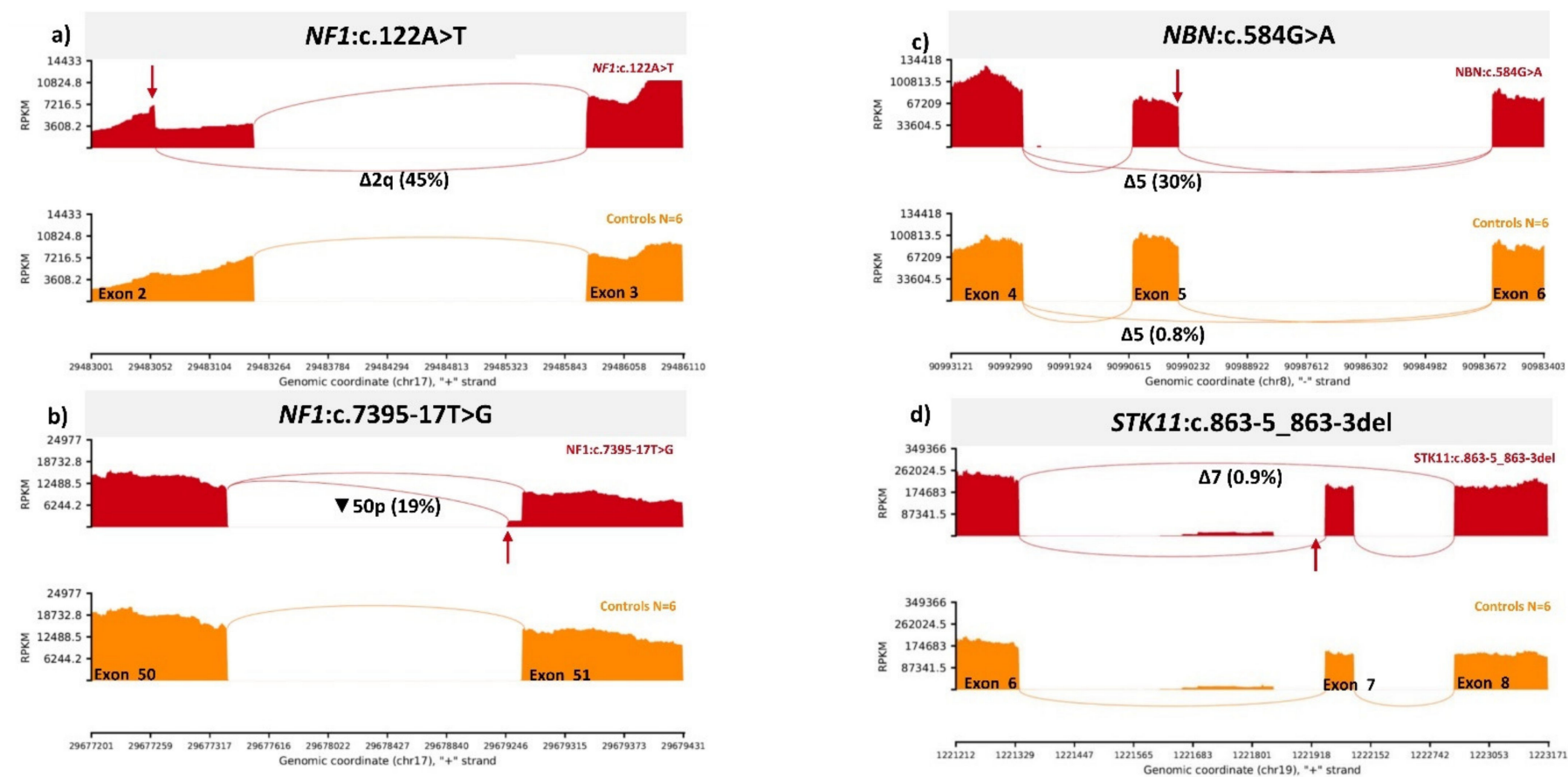

\begin{tabular}{|c|c|c|c|}
\hline cDNA variant & $\begin{array}{l}\text { splicing } \\
\text { event effect on mRNA splicing } \\
\text { description }\end{array}$ & predicted protein & $\begin{array}{l}\text { percentage of aberrant } \\
\text { transcript determined } \\
\text { by RNAseq } \\
\text { (patients / mean } \\
\text { controls) }\end{array}$ \\
\hline
\end{tabular}

\begin{tabular}{|c|c|c|c|c|}
\hline \multirow[b]{2}{*}{$N F 1: c .122 A>T$} & \multirow[b]{2}{*}{$\Delta 2 q$} & \multirow[b]{2}{*}{ r.121_204del } & \\
\hline & & & p.(Glu41_Met68del) & $45 \% / 0 \%$ \\
\hline$N F 1: c .7395-17 T>G$ & $\mathbf{\nabla} 50 \mathrm{p}$ & r.7394_7395ins7395-16_7395-1 & p.(Arg2465Serfs*21) & $19 \% / 0 \%$ \\
\hline NBN:C.584G>A & $\Delta 5$ & r.481_584del & p.(Thr161Phefs*6) & $30 \% / 0.8 \%$ \\
\hline STK11:c.863-5_863-3del & $\Delta 7$ & r.863_920del & p. (Gly288Alafs*29) & $1.1 \% / 0 \%$ \\
\hline STK11:C.615G $>A$ & $\Delta 5 p$ & r.598_616del & p. (Ala200Argfs*81) & $0.9 \% / 0 \%$ \\
\hline
\end{tabular}

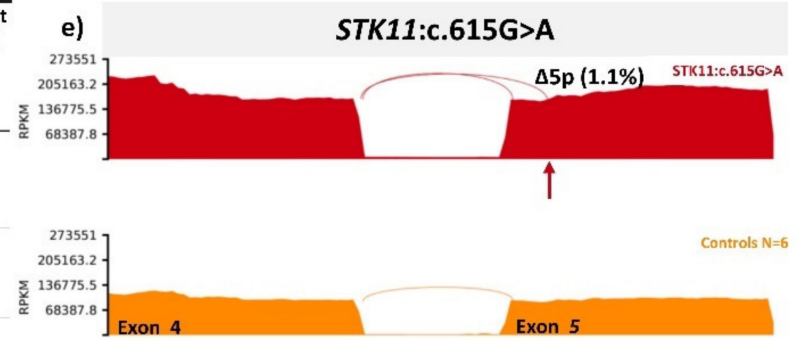

Figure 3. Sashimi plots, representing the splicing impairment caused by variant (a) NF1:c.122A $>T$ (b) NF1:c.7395-17T $>$ G (c) NBN:c.584G>A p.(Ser195Asn) (d) STK11:c.863-5_863-3delCTC (e) STK11:c.615G>A p.(Ala205=). Red arrows label variant's location. Red sashimi plots represent carriers. Orange plots represent control samples $(N=6)$. Junctions without label are normal transcripts; junctions with labels represent aberrant transcripts.

\subsection{Determination of Spliceogenicity of VUS}

Three variants in NBN or STK11 genes, which were bioinformatically predicted to cause splicing impairment, were selected for the analysis: NBN:c.584G>A, STK11:c.8635_863-3delCTC and STK11:c.615G>A. The results are visualized in Figure 3.

$N B N$ :c.584G $>A$ is located in the ultimate position of exon 5, which is predicted to completely abolish natural donor splice site. We observed strengthening of out-of-frame exon 5 skipping $(\Delta 5)$ in the variant carrier. Analyzing the junction data, $\Delta 5$ was present in $30 \%$ of junctions in NBN:c.584G>A carrier in comparison to $0.8 \%$ in controls. After inspecting the alignment file at the position c.584, only wild-type nucleotide $\mathrm{G}$ was detected, implicating that the NBN:c.584G>A variant does not form any full-length transcript.

STK11:c.863-5_863-3delCTC variant is located in intron 6 and is predicted to decrease the strength of native acceptor splice site. RNAseq analysis has revealed an abnormal transcript that lacks exon $7(\Delta 7)$. However, the out-of-frame $\Delta 7$ transcript was minorly expressed (only in 0.9\%), implying that STK11:c.863-5_863-3delCTC variant causes low leaky splicing abnormality. The control samples did not harbor $\Delta 7$ transcript.

Similarly, STK11:c.615G >A creates minor splicing defect (1.1\%) by introducing a de novo acceptor splice site. The variant causes minor frameshift deletion of first $19 \mathrm{nu}$ cleotides of exon $5(\Delta 5 p)$. When inspecting mapped data, mutated A nucleotide at the 
position c. 615 was present in $49 \%$ of the reads, which confirms that the splicing abnormality is minor. The $\Delta 5 \mathrm{p}$ transcript was not present in the controls.

\section{Discussion}

RNA-based experiments are often performed in diagnostics laboratories in order to identify variants that cause RNA splicing impairment [19-22]. RT-PCR followed by capillary electrophoresis and Sanger sequencing are golden standards for determining variants spliceogenicity. However, such experiments are limited to the location of the variant, requiring multiple PCR reactions for different variants in the same gene. Any laboratory, no matter how big or small, requires a reliable straightforward method to determine variants effect on splicing for precise variant classification. Here, we present a simple RNAseq approach that efficiently detects splicing junctions, which can be implemented by any laboratory with an access to an NGS instrument.

The first aim of our study was to evaluate if our pipeline is sensitive enough to detect previously determined naturally occurring alternative splicing events of STK11 gene. We were able to detect $95 \%$ of all splicing events and furthermore detect 18 previously undetected splicing events. One previously described event $\Delta 7$ was not detected by our analysis in the control samples included in the study [9]. Nevertheless, we were able to detect the identical event $\Delta 7$ in a patient with a rare leaky splice-site variant located in intron 6 of STK11 gene (STK11:c.863-5_863-3delCTC) demonstrating that the assay and the pipeline are able to detect such a splicing event, but the transcript was not present in our data. An additional previously described transcript that was not present in our dataset was $\Delta 2-5$. These two events may be population specific, as they were detected in all four samples in a study by Brandão et al., 2019, but were not seen in a single sample in our study (Slovenian population). Notably, we were able to detect all types of splicing events: exon skipping, multiple exon skipping, donor/acceptor shift, cryptic exon inclusion and mixed splicing events. Once the method was established, we characterized alternative splicing patterns of the NBN gene. This is the first time, to our knowledge, that the naturally occurring splicing events of $N B N$ gene were characterized in depth. Ten previously identified alternative splicing events were also detected by our approach [15-17]. This catalogue of alternatively spliced transcripts (Table 2) is an important asset for further characterization of possible spliceogenic variants. Interestingly, exons 8 (amino acids 299-332), 10 and 11 (amino acids 375-615) were not subjected to alternative exon skipping. It might be that these exons are essential for protein function. Indeed, the nibrin protein was shown to interact with mTOR/Rictor/SIN1 complex at the amino acid residues 221-402 [23].

Crucial for any RNAseq experiment is its ability to accurately detect splicing impairment [24]. To test that our assay can detect abnormal splicing, two well-studied splice altering NF1 variants were examined. Our assay correctly identified abnormal splicing junctions that arose due to damaging NF1:c.122A $>$ T and NF1:c.7395-17T $>$ G variants. Moreover, the percentage of aberrant transcript determined by $\mathrm{CE}$ and RNAseq was similar for both variants, meaning the novel RNAseq method can reliably quantify the aberrant versus normal transcript. NF1:c.7395-17T>G variant produced 19\% of aberrant transcript, which might be due to partial degradation of truncated mRNA by nonsense-mediated decay pathway.

Additionally, our method was used to determine splicoegenicity of three variants of uncertain significance. One variant NBN:c.584G $>$ A was shown to completely disturb mRNA splicing by inducing out-of-frame exon 5 skipping and was therefore reclassified as likely pathogenic (ACMG / AMP criteria applied: PS3, PM2, PP3). Two variants STK11:c.8635_863-3delCTC and STK11:c.615G>A were determined to cause minor leaky splicing, as they were expressed in extremely low fractions. Moreover, the carriers of both variants did not have any clinical characteristics of Peutz-Jeghers syndrome, which is caused by STK11 pathogenic variants. Both variants were therefore reclassified as likely benign (ACMG/AMP criteria applied: BS3, BP5, PM2). 
Here, we show that our assay can indeed detect altered transcripts, both complete splicing aberrations and leaky splicing, and can be therefore used as a complementary test in molecular diagnostics laboratories to characterize variants' effect on splicing. Our approach might be used as an alternative method to targeted RNA sequencing or whole transcriptome sequencing when examining one gene of interest.

The main advantage of our approach compared with the targeted RNAseq is that there is no need to design enrichment probes. Designing enrichment probes can be challenging especially for poorly researched genes $[25,26]$. Furthermore, targeted RNAseq assays are frequently designed and validated for a specific gene panel, which makes it difficult to add or remove genes of interest [26]. An additional advantage of our assay in comparison to targeted or transcriptome sequencing is the possibility to study a single gene of interest, achieving a higher coverage crucial for detection of events expressed in lower fractions. Our assay can be customized for nearly any gene of interest, which makes it highly suitable for laboratories dealing with rare genetic syndromes. The method is limited to fresh tissue samples or cell cultures, since the RNA has to be of high quality so that it is amplifiable with long-range PCR. An additional limitation is the size of the CDNA, which needs to be amplifiable with PCR. In this study, the maximal length of cDNA, which was successfully amplified and analyzed, was 12kb (NF1 gene).

The limitation of our approach in detecting natural events of NBN gene was that DNA sequencing of control samples only included exon regions and $25 \mathrm{bp}$ of intronic sequence. Although deep intronic variants were not ruled out, we avoided rare genetic variants by only counting junctions detected in two or more control samples. Additionally, the newly detected splicing events in our study were not confirmed with an alternative method. To further improve our protocol, molecular barcodes could be used in order to partially avoid biased amplification of certain splicing events. An additional drawback of this study is the low number of samples used for the catalogue of natural splicing events. Moreover, the catalogue of naturally occurring splicing isofroms was conducted with shortread sequencing, which may cause mapping errors. The catalogue could be improved by confirming the isoforms with long-read sequencing, such as single-molecule real-time or nanopore sequencing. Importantly, with our approach, we are only able to determine splicing events not the whole full-length splicing isoforms, which can be achieved with long-read sequencing.

\section{Conclusions}

In conclusion, our novel single-gene assay is a fast, straightforward and cost-efficient technique for discovering splicing impairment. All that a laboratory requires is a set of primers that align to the $5^{\prime}$ and $3^{\prime} \mathrm{UTR}$ region of the gene of interest, long-range PCR amplification, Nextera XT library preparation kit and access to an NGS instrument. RNAseq data can be analyzed on any desktop computer, without the need for high computational power. Low sequencing cost and low computational power for data analysis make the method accessible even to laboratories with limited budgets. The turnaround time starting from RNA isolation to loading a sequencing library onto an NGS instrument is around $10 \mathrm{~h}$ (the duration of the experiment depends on the length of cDNA that needs to be amplified with PCR). Our method can be easily adopted in diagnostics laboratories, as the assay can be performed in a time frame necessary for clinical testing. Importantly, when the method is applied in the clinical laboratory, it should always include control samples for excluding normally present splicing events in the diagnostics sample.

Supplementary Materials: The following are available online at https:/ / www.mdpi.com/article/ 10.3390/biology10080706/s1, Table S1: naturally occurring splicing junctions of NBN and STK11 genes in the blood of six control samples, obtained with RNAseq and STAR aligner; Table S2: primer sequences and PCR protocol used for long-range amplification of NBN, STK11 and NF1 genes. Benign variants detected in NBN and STK11 genes in six controls with DNAseq. 
Author Contributions: Conceptualization, V.Š.D. and S.N.; methodology, V.Š.D.; software, V.Š.D.; validation, V.Š.D.; formal analysis, V.Š.D.; investigation, V.Š.D., S.N., G.K., A.B., V.S. and M.K.; writing—original draft preparation, V.Š.D.; writing—review and editing, S.N.; visualization, V.Š.D.; supervision, S.N.; funding acquisition, S.N. All authors have read and agreed to the published version of the manuscript.

Funding: The study was funded from Slovenian research agency, program number: P3-0352.

Institutional Review Board Statement: The study was conducted according to the guidelines of the Declaration of Helsinki, and approved by the Institutional Review Board (or Ethics Committee) of the Institute of Oncology Ljubljana (permission no. OIRIEK00798, 18.6.2019) and by the National Medical Ethics Committee of Republic of Slovenia (permission no. 0120-339/2019/5, date of approval: 16 July 2019).

Informed Consent Statement: Informed consent was obtained from all subjects involved in the study. Written informed consent was obtained from the patient(s) to publish this paper.

Data Availability Statement: The data presented in this study are available upon request from the corresponding author. The data are not publicly available due to patients' privacy.

Acknowledgments: Our sincere thanks to the patients for their participation. We also thank Aleksander Novaković for bioinformatic support and Katarina Gimpelj for laboratory assistance.

Conflicts of Interest: The authors declare no conflict of interest.

\section{References}

1. Colombo, M.; Blok, M.J.; Whiley, P.; Santamariña, M.; Gutiérrez-Enríquez, S.; Romero, A.; Garre, P.; Becker, A.; Smith, L.D.; De Vecchi, G.; et al. Comprehensive annotation of splice junctions supports pervasive alternative splicing at the BRCA1 locus: A report from the ENIGMA consortium. Hum. Mol. Genet. 2014, 23, 3666-3680. [CrossRef] [PubMed]

2. Yamada, M.; Suzuki, H.; Shiraishi, Y.; Kosaki, K. Effectiveness of integrated interpretation of exome and corresponding transcriptome data for detecting splicing variants of genes associated with autosomal recessive disorders. Mol. Genet. Metab. Rep. 2019, 21, 100531. [CrossRef] [PubMed]

3. Cartegni, L.; Chew, S.L.; Krainer, A.R. Listening to silence and understanding nonsense: Exonic mutations that affect splicing. Nat. Rev. Genet. 2002, 3, 285-298. [CrossRef]

4. Karam, R.; Conner, B.; LaDuca, H.; McGoldrick, K.; Krempely, K.; Richardson, M.E.; Zimmermann, H.; Gutierrez, S.; Reineke, P.; Hoang, L.; et al. Assessment of Diagnostic Outcomes of RNA Genetic Testing for Hereditary Cancer. JAMA Netw. Open 2019, 2, e1913900. [CrossRef]

5. Whiley, P.J.; De La Hoya, M.; Thomassen, M.; Becker, A.; Brandão, R.; Pedersen, I.S.; Montagna, M.; Menéndez, M.; Quiles, F.; Gutiérrez-Enríquez, S.; et al. Comparison of mRNA splicing assay protocols across multiple laboratories: Recommendations for best practice in standardized clinical testing. Clin. Chem. 2014, 60, 341-352. [CrossRef] [PubMed]

6. Kulkarni, P.; Frommolt, P. Challenges in the Setup of Large-scale Next-Generation Sequencing Analysis Workflows. Comput. Struct. Biotechnol. J. 2017, 15, 471-477. [CrossRef]

7. Ozsolak, F.; Milos, P.M. RNA sequencing: Advances, challenges and opportunities. Nat. Rev. Genet. 2011, 12, 87-98. [CrossRef]

8. Davy, G.; Rousselin, A.; Goardon, N.; Castéra, L.; Harter, V.; Legros, A.; Muller, E.; Fouillet, R.; Brault, B.; Smirnova, A.S.; et al. Detecting splicing patterns in genes involved in hereditary breast and ovarian cancer. Eur. J. Hum. Genet. 2017, 25, 1147-1154. [CrossRef]

9. Brandão, R.D.; Mensaert, K.; López-Perolio, I.; Tserpelis, D.; Xenakis, M.; Lattimore, V.; Walker, L.C.; Kvist, A.; Vega, A.; Gutiérrez-Enríquez, S.; et al. Targeted RNA-seq successfully identifies normal and pathogenic splicing events in breast/ovarian cancer susceptibility and Lynch syndrome genes. Int. J. Cancer 2019, 145, 401-414. [CrossRef]

10. Setrajcic Dragos, V.; Blatnik, A.; Klancar, G.; Stegel, V.; Krajc, M.; Blatnik, O.; Novakovic, S. Two novel NF1 pathogenic variants causing the creation of a new splice site in patients with neurofibromatosis type I. Front. Genet. 2019, 10, 762. [CrossRef]

11. Klančar, G.; Blatnik, A.; Šetrajčič Dragoš, V.; Vogrič, V.; Stegel, V.; Blatnik, O.; Drev, P.; Gazič, B.; Krajc, M.; Novaković, S. A Novel Germline MLH1 In-Frame Deletion in a Slovenian Lynch Syndrome Family Associated with Uncommon Isolated PMS2 Loss in Tumor Tissue. Genes (Basel) 2020, 11, 325. [CrossRef] [PubMed]

12. Dobin, A.; Gingeras, T.R. Mapping RNA-seq Reads with STAR. Curr. Protoc. Bioinform. 2015, 51, 11-14. [CrossRef] [PubMed]

13. Li, H.; Handsaker, B.; Wysoker, A.; Fennell, T.; Ruan, J.; Homer, N.; Marth, G.; Abecasis, G.; Durbin, R. The Sequence Alignment/Map format and SAMtools. Bioinformatics 2009, 25, 2078-2079. [CrossRef]

14. Jaganathan, K.; Kyriazopoulou Panagiotopoulou, S.; McRae, J.F.; Darbandi, S.F.; Knowles, D.; Li, Y.I.; Kosmicki, J.A.; Arbelaez, J.; Cui, W.; Schwartz, G.B.; et al. Predicting Splicing from Primary Sequence with Deep Learning. Cell 2019, 176, 535-548.e24. [CrossRef] [PubMed]

15. Varon, R.; Dutrannoy, V.; Weikert, G.; Tanzarella, C.; Antoccia, A.; Stöckl, L.; Spadoni, E.; Krüger, L.-A.; di Masi, A.; Sperling, K.; et al. Mild Nijmegen breakage syndrome phenotype due to alternative splicing. Hum. Mol. Genet. 2006, 15, 679-689. [CrossRef] 
16. Tessitore, A.; Biordi, L.; Flati, V.; Toniato, E.; Marchetti, P.; Ricevuto, E.; Ficorella, C.; Scotto, L.; Giannini, G.; Frati, L.; et al. New mutations and protein variants ofNBS1 are identified in cancer cell lines. Genes Chromosom. Cancer 2003, 36, 198-204. [CrossRef] [PubMed]

17. Takakuwa, T.; Luo, W.-J.; Francisca Ham, M.; Aozasa, K. A 50-bp insertion from intron 2 between exons 2 and 3 ofNBS1 may be a spliced variant. Genes, Chromosom. Cancer 2004, 39, 341-342. [CrossRef]

18. Mayr, C. Evolution and Biological Roles of Alternative 3'UTRs. Trends Cell Biol. 2016, 26, 227-237. [CrossRef] [PubMed]

19. Landrith, T.; Li, B.; Cass, A.A.; Conner, B.R.; LaDuca, H.; McKenna, D.B.; Maxwell, K.N.; Domchek, S.; Morman, N.A.; Heinlen, C.; et al. Splicing profile by capture RNA-seq identifies pathogenic germline variants in tumor suppressor genes. Npj Precis. Oncol. 2020, 4, 4. [CrossRef]

20. Farber-Katz, S.; Hsuan, V.; Wu, S.; Landrith, T.; Vuong, H.; Xu, D.; Li, B.; Hoo, J.; Lam, S.; Nashed, S.; et al. Quantitative Analysis of BRCA1 and BRCA2 Germline Splicing Variants Using a Novel RNA-Massively Parallel Sequencing Assay. Front. Oncol. 2018, 8, 286. [CrossRef]

21. Casadei, S.; Gulsuner, S.; Shirts, B.H.; Mandell, J.B.; Kortbawi, H.M.; Norquist, B.S.; Swisher, E.M.; Lee, M.K.; Goldberg, Y.; O'Connor, R.; et al. Characterization of splice-altering mutations in inherited predisposition to cancer. Proc. Natl. Acad. Sci. USA 2019, 116, 26798-26807. [CrossRef]

22. Krivokuca, A.; Dragos, V.S.; Stamatovic, L.; Blatnik, A.; Boljevic, I.; Stegel, V.; Rakobradovic, J.; Skerl, P.; Jovandic, S.; Krajc, M.; et al. Novel BRCA1 splice-site mutation in ovarian cancer patients of Slavic origin. Fam. Cancer 2018, 17, 179-185. [CrossRef] [PubMed]

23. Wang, J.-Q.; Chen, J.-H.; Chen, Y.-C.; Chen, M.-Y.; Hsieh, C.-Y.; Teng, S.-C.; Wu, K.-J. Interaction between NBS1 and the mTOR/Rictor/SIN1 Complex through Specific Domains. PLoS ONE 2013, 8, e65586. [CrossRef] [PubMed]

24. Houdayer, C.; Caux-Moncoutier, V.; Krieger, S.; Barrois, M.; Bonnet, F.; Bourdon, V.; Bronner, M.; Buisson, M.; Coulet, F.; Gaildrat, P.; et al. Guidelines for splicing analysis in molecular diagnosis derived from a set of 327 combined in silico/in vitro studies on BRCA1 and BRCA2 variants. Hum. Mutat. 2012, 33, 1228-1238. [CrossRef]

25. Kukurba, K.R.; Montgomery, S.B. RNA Sequencing and Analysis. Cold Spring Harb. Protoc. 2015, 2015, 951-969. [CrossRef]

26. Hrdlickova, R.; Toloue, M.; Tian, B. RNA-Seq methods for transcriptome analysis. Wiley Interdiscip. Rev. RNA 2017, 8, e1364. [CrossRef] [PubMed] 